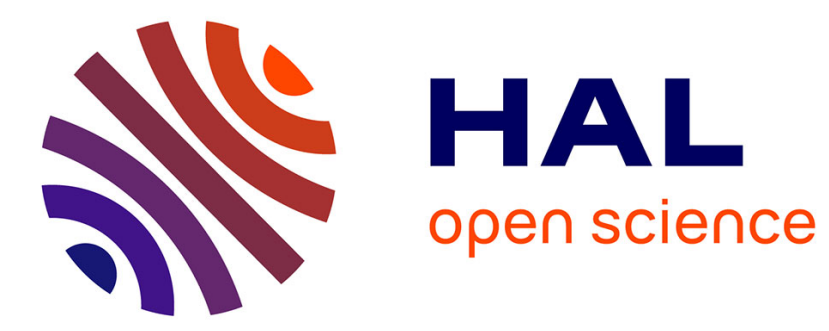

\title{
Residual Doping in Homoepitaxial Zinc Oxide Layers Grown by Metal Organic Vapor Phase Epitaxy
}

Isabelle Bisotto, Carole Granier, Stéphane Brochen, Alexandre Ribeaud, Pierre Ferret, Gauthier Chicot, Johan Rothman, Julien Pernot, Guy Feuillet

\section{- To cite this version:}

Isabelle Bisotto, Carole Granier, Stéphane Brochen, Alexandre Ribeaud, Pierre Ferret, et al.. Residual Doping in Homoepitaxial Zinc Oxide Layers Grown by Metal Organic Vapor Phase Epitaxy. Japanese Journal of Applied Physics, part 2 : Letters, 2010, 3 (9), pp.095802. 10.1143/APEX.3.095802 . hal00734732

\section{HAL Id: hal-00734732 \\ https://hal.science/hal-00734732}

Submitted on 24 Sep 2012

HAL is a multi-disciplinary open access archive for the deposit and dissemination of scientific research documents, whether they are published or not. The documents may come from teaching and research institutions in France or abroad, or from public or private research centers.
L'archive ouverte pluridisciplinaire HAL, est destinée au dépôt et à la diffusion de documents scientifiques de niveau recherche, publiés ou non, émanant des établissements d'enseignement et de recherche français ou étrangers, des laboratoires publics ou privés. 


\title{
Residual Doping in Homoepitaxial Zinc Oxide Layers Grown by Metal Organic Vapor Phase Epitaxy
}

\author{
Isabelle Bisotto, Carole Granier, Stephane Brochen, Alexandre Ribeaud, Pierre Ferret, \\ Gauthier Chicot, Johan Rothman, Julien Pernot ${ }^{1}$, and Guy Feuillet*
}

\author{
CEA-LETI, Minatec, 17 rue des Martyrs, 38054 Grenoble cedex 9, France \\ ${ }^{1}$ Institut NEEL, CNRS and Université Joseph Fourier, BP166, 38042 Grenoble Cedex 9, France
}

Received July 6, 2010; accepted August 15, 2010; published online September 3, 2010

Full maximum entropy mobility spectrum analysis was carried out on the basis of temperature and magnetic-field-dependent Hall measurements to assess the transport properties of homoepitaxial metal organic vapor phase epitaxy zinc oxide layers. Two different conductivity channels were clearly identified and the channel with higher mobility and higher carrier concentration is associated with the epitaxial layer. Hydrogen impurity acting as residual donor and as a passivating species for acceptors is proposed to explain the higher carrier concentration and mobility in the epilayer. In contrast to heteroepitaxial layers, no conduction channel is observed from the substrate to epilayer interface.

(C) 2010 The Japan Society of Applied Physics

$\mathbf{Z}$ $\mathrm{nO}$ is an appealing II-VI semiconductor material for light emitters in the blue-to-UV range, because of its $3.37 \mathrm{eV}$ direct band gap and large exciton binding energy $(60 \mathrm{meV})$. One of the challenges currently being addressed by the $\mathrm{ZnO}$ community is obtaining epitaxial thin films with reliable and stable p-type conductivity. p-type doping is limited by the existence of acceptor killers such as the intrinsic or extrinsic impurities. On the other hand, structural defects, if present in the material, can also make it difficult to control doping. ${ }^{1,2)}$ The electrical properties of nonintentionally doped $\mathrm{ZnO}$ thin films grown by metalorganic vapour phase epitaxy (MOVPE) on sapphire substrate were investigated by Pagni et al. ${ }^{3)}$ By applying the two-layer model ${ }^{4)}$ to take account of the interfacial conductivity contribution, the authors showed on one hand that the conductivity and the carrier activation energy of the MOVPE grown films depended on the II-VI ratio, and on the other hand, the presence of an interface conductivity between the substrate and the epilayer. The high density of defects present in the heteroepitaxially grown layers may cast a doubt on the intrinsic nature of the donors suggested to be at the origin of the conductivity. Thus it is important, in order to control the doping of the epilayer, to assess the transport properties from the epilayer independently of that of the substrate and from that of the interface.

In order to shed some light on this subject, Hall effect measurements were carried out as a function of temperature $(50$ to $400 \mathrm{~K})$ and magnetic field $(0-9 \mathrm{~T})$ in order to investigate the electrical properties of nonintentionally doped MOVPE $\mathrm{ZnO}$ thin film homoepitaxially grown on O-face $\mathrm{ZnO}$ substrate from Crystec (hydrothermal synthesis) with a low dislocation density $\left(\leq 10^{5} \mathrm{~cm}^{-2}\right)$.

The 500-nm-thick epilayer was grown in a horizontal hot wall MOVPE system, at a temperature of $950^{\circ} \mathrm{C}$ and under a pressure of $50 \mathrm{mbar}$, using $\mathrm{N}_{2} \mathrm{O}$ and diethyl zinc (DeZn) as precursors for $\mathrm{O}$ and $\mathrm{Zn}$, respectively, onto $\mathrm{O}$-face hydrothermal $\mathrm{ZnO}$ substrate. Prior to growth, a soft surface finish was carried out using a chemical-mechanical polishing (CMP) process followed by a high-temperature annealing at $1100{ }^{\circ} \mathrm{C}$ under oxygen. The inlet VI to II ratio was maintained at 25000 , which led to a growth rate of $150 \mathrm{~nm} \cdot \mathrm{h}^{-1}$. The (0002) X-ray rocking curve did not show

*E-mail address: guy.feuillet@cea.fr any epilayer-related widening of the diffraction peaks compared with the substrate, indicating that the structural quality of the 500-nm-thick epilayer is at least as good as that of the substrate (the X-ray penetration depth being of the order of $5 \mu \mathrm{m}$ ).

Two samples (samples 1 and 2) were cut out from the 500-nm-thick epilayer grown on the hydrothermal $\mathrm{ZnO}$ substrate. Sample 1 was CMP-polished in order to remove the epilayer from the substrate. Sample 2 was left un-etched with the epilayer on the top. Hall effect and resistivity measurements were carried out on the two samples using van der Pauw patterns in the temperature range from 50 to $400 \mathrm{~K}$ and for magnetic fields from 0 to $9 \mathrm{~T}$. For ohmic contacts, indium pads were soldered on the four corners of the sample but were not annealed. The full maximum entropy mobility spectrum analysis (f-MEMSA) described in ref. 5, which we used formerly to differentiate between bulk and surface conductivity in $\mathrm{ZnO}$ substrates, ${ }^{6}$ was implemented to analyze the magnetic-field and temperaturedependent Hall effect results. From this algorithm, one can extract the different mobility contributions to the conductivity spectrum, related to the substrate, the $\mathrm{ZnO}$ thin film itself, and the possible substrate-to-film interface with no assumption on the number of conductivity channels, contrary to a multilayer model where the number of channels is predetermined.

Capacitance-voltage measurements at $1 \mathrm{MHz}$ were carried out on sample 2 to measure the active donor concentration $\left[N_{\mathrm{D}}-N_{\mathrm{A}}\right]_{C(V)}$ in the MOVPE layer and compare it with the result of the f-MEMSA analysis.

Figure 1 shows the raw data for the Hall mobility and sheet carrier density for samples 1 and 2 . From 50 to $200 \mathrm{~K}$, the mobility of sample 2 (substrate with epilayer) is higher than that of sample 1 (substrate after removal of the epilayer), probably due to the epilayer contribution. The mobility of sample 1 is commonly obtained for hydrothermal substrates after annealing in $\mathrm{O}_{2}$ at $1100^{\circ} \mathrm{C} .{ }^{6}$ ) In terms of sheet carrier density, no direct evidence of the presence of the epilayer is observed, Hall data of samples 1 and 2 are superposed.

In view of these compared Hall results, the f-MEMSA was used. Generally, the discrepancies between the samples and the ideal semiconductor model used with the f-MEMSA can limit the power of the algorithm to separate two 


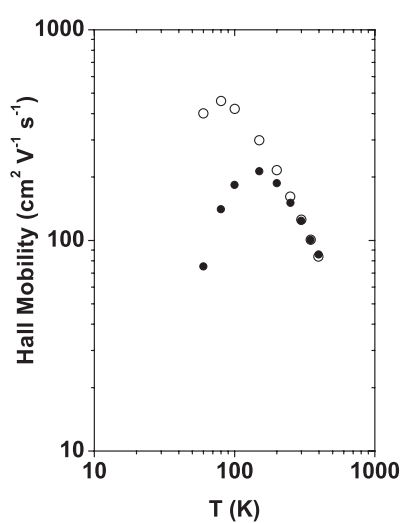

(a)

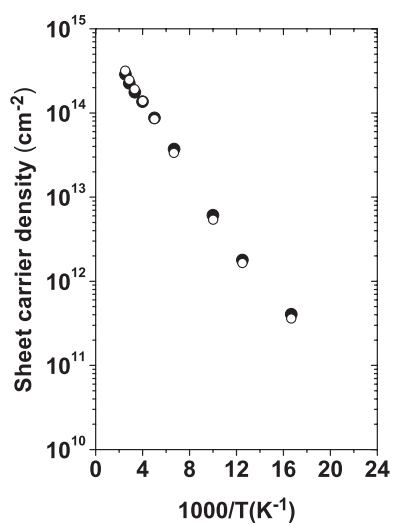

(b)
Fig. 1. Temperature dependence of (a) Hall mobility and (b) sheet carrier density of sample 1 (closed circles: after epilayer removal) and sample 2 (open circles: before epilayer removal).

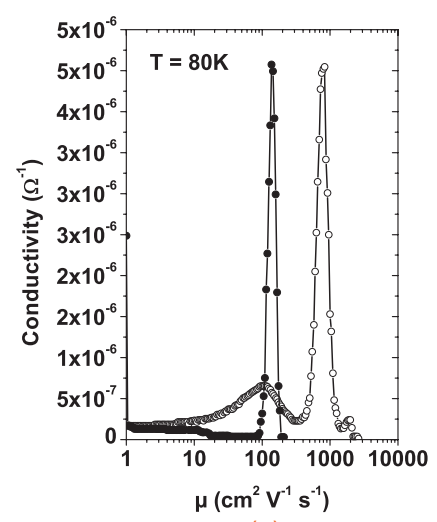

(a)

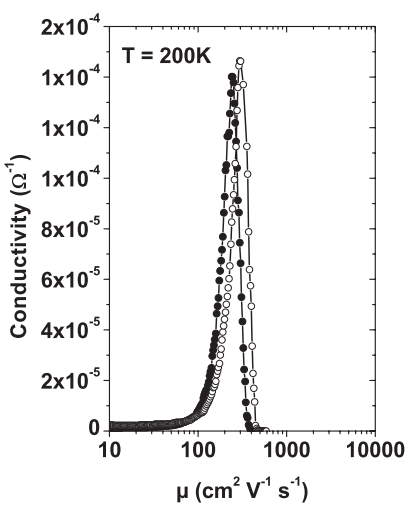

(b)
Fig. 2. Conductivity spectrum at 80 and $200 \mathrm{~K}$ obtained by f-MEMSA for sample 1 (closed circles: after epilayer removal) and sample 2 (open circles: before epilayer removal).

contributions with similar mobilities. This limits the precision of the analysis at high temperature (typically $\geq 200 \mathrm{~K}$ ) due to the phonon scattering mechanism that dominates the mobility, leading to almost the same mobility values for the different kinds of materials as shown in Fig. 2(b). Consequently, the number of conductivity channels is only determined at low temperatures. In Fig. 2(a), at $80 \mathrm{~K}$, two different electronic mobility contributions to the conductivity for sample 2 (substrate with epilayer) are observed, whereas a single one is detected for sample 1 (substrate after epilayer removal). We want to point out that artefact contributions (a broadened conduction peak of the opposite type of carriers, namely, holes), exist but are not represented on Fig. 2. As discussed in refs. 5 and 6, this effect can have different origins: the size effects, the geometry of the contacts in the van der Pauw configuration, and the inhomogeneity of the sample. In addition, many $\mathrm{ZnO}$ samples showed negative magnetoresistance (MR) at low temperatures, which suggests the presence of disordered magnetic scattering in the samples. This effect is not described using the MEMSA model and this would also lead to errors and artifacts in the estimated spectra. However, in the present samples, negative MR was only

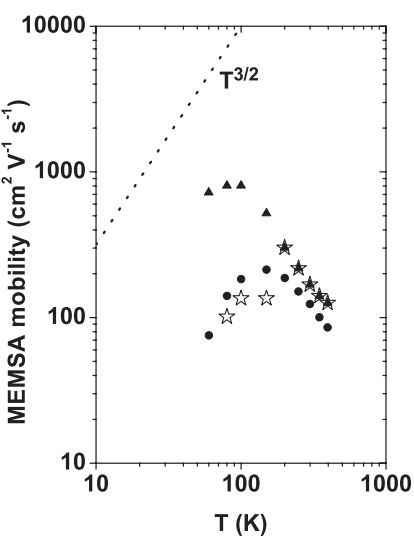

(a)

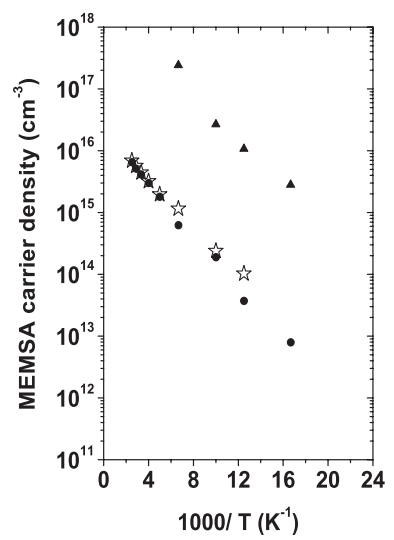

(b)
Fig. 3. Temperature dependence of f-MEMSA (a) mobility and (b) carrier density for sample 1 (closed circles: after epilayer removal) and for the two channels of sample 2 (triangles and stars: before epilayer removal). The thicknesses used to calculate the carrier density are $d \sim 500 \mathrm{~nm}$ and $d \sim 500 \mu \mathrm{m}$, respectively, for the epilayer and the substrate. The dashed line shows a $T^{3 / 2}$ temperature dependence corresponding to ionized impurity scattering.

observed at the lowest temperature, typically $<100 \mathrm{~K}$, where this perturbation can be expected to be low.

The carrier densities and mobilities obtained from the fMEMSA analysis over the entire temperature range, before (sample 2) and after layer removal (sample 1) are shown in Fig. 3. As discussed above, at higher temperatures, the different conductivity channels merge due to phonon interactions, but upon decreasing the temperature, the two mobility components of sample 2 appear. One of these is very close to the one obtained for sample 1 , also represented in Fig. 3, and thus could reasonably be attributed to the substrate. Consequently, the second component could be that of the epilayer itself. At low temperature, ionized impurity scattering is the major contribution to the mobility as illustrated by the $T^{3 / 2}$ temperature dependence line on the graph, whereas at high temperature, it is less straightforward and results from mixed phonon interactions. The analysis of the carrier density leads to the same activation energy of $\sim 30 \mathrm{meV}$ for the two channels of sample 2 and for the one and only channel of sample 1 , and corresponds to that of annealed hydrothermal Crystec substrates as measured by Tavares et al. ${ }^{6)}$ For sample 1 (after epilayer removal), the fit of the temperature dependence of the carrier concentration by the neutrality equation yields a compensation ratio $N_{\mathrm{A}} / N_{\mathrm{D}}$ of the order of 0.01 . The carrier densities at $100 \mathrm{~K}$ are $\sim 2 \times 10^{16} \mathrm{~cm}^{-3}$ for the MOVPE thin film and $\sim 2 \times$ $10^{14} \mathrm{~cm}^{-3}$ for the substrate, in the range of the value usually obtained for annealed substrates. ${ }^{6,7)} C-V$ analysis carried out on sample 2 yielded an active donor concentration $\left[N_{\mathrm{D}}-N_{\mathrm{A}}\right]=10^{17} \mathrm{~cm}^{-3}$. A strict comparison with the results obtained from the f-MEMSA analysis is not possible due to the uncertainty in the evaluation of free carrier concentrations at high temperatures, but at least the two analyses do not contradict each other.

Among the extrinsic impurities, only lithium and hydrogen are supposed to give rise to shallow levels ${ }^{8)}$ and could explain the low activation energy measured. From secondary ion mass spectrometry, (SIMS) analysis carried out on the 
same epilayer (not shown here), lithium was found at a lower concentration in the layer (i.e., $\sim 10^{15} \mathrm{~cm}^{-3}$ ) than in the substrate $\left(\sim 10^{17} \mathrm{~cm}^{-3}\right)$, which rules out any hypothesis based on the diffusion of Li during growth. For hydrogen, which could have been incorporated as a result of DeZn decomposition or due to out-diffusion from the substrate, ${ }^{9)}$ SIMS indicates a higher concentration in the layer, just above the high detection limit of $\sim 10^{18} \mathrm{~cm}^{-3}$. Thus, hydrogen, as an interstitial or substitutional (Ho) donor or possibly through donor-type complex formation ${ }^{10)}$ could be responsible for the rather high $\mathrm{n}$ type doping of the homoepitaxial MOVPE. Furthermore, hydrogen could also passivate acceptors present in the layer making them electrically inactive ${ }^{11)}$ with a subsequent reduction of the compensation ratio.

On the other hand, the electron mobility in the MOVPE thin film is about $800 \mathrm{~cm}^{2} \mathrm{~V}^{-1} \mathrm{~s}^{-1}$ at $100 \mathrm{~K}$ compared with about $200 \mathrm{~cm}^{2} \mathrm{~V}^{-1} \mathrm{~s}^{-1}$ for the substrate despite a similar structural quality [Fig. 3(a)]. In this case too, the lower compensation of the epilayer compared with the substrate could be the origin of the observed difference. Indeed, at low temperature, electron scattering by ionized impurities is more important in the substrate than in the epilayer [cf. Fig. 3(a)]. The relaxation time due to ionized impurities is proportional to $1 / N_{\mathrm{ii}}$, where $N_{\mathrm{ii}}$ is the density of ionized impurities. $N_{\text {ii }}$ can be approximated to $2 N_{\mathrm{A}}$ for low temperatures. Consequently, the increase of the mobility observed for the epilayer could be the result of a decreased $N_{\mathrm{A}}$.

Another important implication of the study is that no contribution of the MOVPE film/substrate interface to the conductivity is present since no degenerate channel is observed, which would otherwise be characterized by a temperature-independent carrier density.

In conclusion, a temperature and magnetic-field-dependent Hall effect study was undertaken on a $\mathrm{ZnO}$ homo- epitaxial MOVPE-grown thin film. Using the f-MEMSA analysis, two different conductivity channels were identified. The high mobility/high carrier concentration channel was unambiguously attributed to the epilayer. Hydrogen acting as a donor and compensating residual acceptors is proposed to explain the higher carrier concentration in the epilayer and its higher mobility. In contrast to the case of heteroepitaxial growth on sapphire substrates, no layer-to-substrate interface conductivity channel was found, opening the way to homoepitaxially grown layers with a better control of their doping properties.

Acknowledgments We wish to acknowledge the French National Agency for Research (ANR) through funding from the CARNOT Institutes. We also thank Pierre Muret from CNRS/Institut Néel for very fruitful discussions in the course of this work.

1) M. Schirra, R. Schneider, A. Reiser, G. M. Prinz, M. Feneberg, J. Biskupek, U. Kaiser, C. E. Krill, K. Thonke, and R. Sauer: Phys. Rev. B 77 (2008) 125215.

2) A. Krtschil, D. C. Look, Z.-Q. Fang, A. Dadgar, A. Diez, and A. Krost: Physica B 376-377 (2006) 703.

3) O. Pagni, N. N. Somhlahlo, C. Weichsel, and A. W. R. Leitch: Physica B 376-377 (2006) 749.

4) D. C. Look and J. R. Molnar: Appl. Phys. Lett. 70 (1997) 3377.

5) J. Rothman, J. Meilhan, G. Perrais, J. P. Belle, and O. Gravand: J. Electron. Mater. 35 (2006) 1174.

6) C. Chevalier-Tavares, J. Rothman, and G. Feuillet: Mater. Res. Soc. Symp. Proc. 1035E (2007) L04-06.

7) D. C. Look, D. C. Reynolds, J. R. Sizelove, R. L. Jones, C. W. Litton, G. Cantwell, and W. C. Harsch: Solid State Commun. 105 (1998) 399.

8) A. Janotti and C. G. Van de Walle: Rep. Prog. Phys. 72 (2009) 126501.

9) E. V. Monakhov, A. Yu. Kuznetsov, and B. G. Svensson: J. Phys. D 42 (2009) 153001.

10) H. Qiu, B. Meyer, Y. Wang, and C. Wöll: Phys. Rev. Lett. 101 (2008) 236401.

11) D. C. Look, B. Claflin, and H. E. Smith: Appl. Phys. Lett. 92 (2008) 122108. 